\title{
DETECTION OF ACANTHAMOEBA SPP. FROM DUST PHENOMENON IN ILAM PROVINCE, WEST IRAN
}

\author{
REZA SABERI ${ }^{1,2}$, AZAR NAJAFI ${ }^{3,4}$ and RAZI NASERIFAR ${ }^{3 *}$ \\ ${ }^{1}$ Department of Parasitology, School of Medicine, Mazandaran University of Medical \\ Sciences, Sari, Iran \\ ${ }^{2}$ Student Research Committee, Mazandaran University of Medical Science, Sari, Iran \\ ${ }^{3}$ Department of Parasitology, Faculty of Medicine, Ilam University of Medical Sciences, \\ Ilam, Iran \\ ${ }^{4}$ Student Research Committee, Ilam University of Medical Sciences, Ilam, Iran
}

(Received: 3 January 2019; accepted: 18 February 2019)

In recent years, increasing dust phenomenon in the west of Iran has led to social, economic, and health concerns. This study aimed to represent the existence and genotyping of Acanthamoeba spp. in dust phenomenon in Ilam Province, Iran. In this study, 55 dust samples were collected and by targeting the diagnostic fragment 3 region of the $18 \mathrm{~S}$ rRNA gene, the genotypes were determined. Utilizing the tolerance ability test, the pathogenic potential of all positive isolates was also recognized. Eighteen samples of Acanthamoeba (32.7\%) were detected in the sampling areas. According to sequencing analysis, the isolates related to T4 (77.7\%) and T2 (22.3\%) genotypes were reported. It was revealed by thermo- and osmotolerance tests in which six strains are extremely pathogenic. To our knowledge, the pathogenic Acanthamoeba was potentially isolated initially from dust phenomenon in Ilam Province. Thus, these strains are probably highly virulent, and dusts are possible sources of Acanthamoeba infection in humans.

Keywords: Acanthamoeba, genotyping, dust, Ilam, Iran

\section{Introduction}

Acanthamoeba spp. are free-living amoebae dispersed in different ecological environments and isolated from soil, air, dust, recreational and mineral water, and sewage samples [1]. Acanthamoeba spp. include two steps of the trophozoite and the cyst in their life cycle [2]. Trophozoites are slender, with spine-like processes known as acanthopodia, with the diameter of 10-50 $\mu \mathrm{m}$ and they are

\footnotetext{
*Corresponding author; E-mail: razinaserifar@yahoo.com
} 
divided by binary fission. Cysts are wrinkled, double-walled, star-shaped, hexagonal, polygonal, or spherical with the diameter of about 20-25 $\mu \mathrm{m}[2,3]$.

Several strains of Acanthamoeba are the causative agents of Acanthamoeba keratitis (AK), a painful sight-threatening disease of the eyes [4]. They are also a causative agent of granulomatous amoebic encephalitis, which is a severe infection of the central nervous system in immunocompromised people [5]. In addition, these amoebae can act as carrier of pathogenic microorganisms such as bacteria and viruses. Bacteria could remain in amoebae and develop the high virulence and resistance to antibiotic. The results of the previous studies reported a high-level resistance of Legionella pneumophila within Acanthamoeba polyphaga to disinfection and antimicrobials [6, 7].

To date, molecular classification of Acanthamoeba genus based on the $18 \mathrm{~S}$ ribosomal RNA sequence has described 21 genotypes (T1-T21) [8]. Numerous Acanthamoeba genotypes T4 have been reported in literature as common genotype in the environmental and clinical samples and the causative agent of different diseases [9]. Other pathogenic genotypes including T2, T3, T5, T6, T10, T11, T12, T15, and T18 were identified [10]. Khan et al. provided a simple plating assay to differentiate pathogenic isolates from the non-pathogenic. In the mentioned study, amoeba with ability to grow at high temperature and osmolarity seems to be pathogenic amoeba [11].

The dust phenomenon is one of the risks in nature, i.e., climatic-atmospheric disasters, that has caused a major concern in recent years [12]. The dust phenomena exist highly in the arid and semi-arid areas. In recent decade, Ilam Province exposed to dust phenomenon throughout the year and this phenomenon has been in higher severity and length [13]. To our knowledge, this is the first study to assess the presence of Acanthamoeba spp. in dust phenomena collected in Ilam Province.

\section{Material and Methods}

Study areas

This study was conducted from June to September 2017 in Ilam Province $\left(32^{\circ} 03^{\prime}-34^{\circ} 02^{\prime}\right.$ north latitude and $45^{\circ} 40^{\prime}-48^{\circ} 03^{\prime}$ east longitude), located in the west of Iran, with the same borders with Khuzestan Province in the south, Lorestan Province in the east, Kermanshah Province in the north and with the same borders with Iraq in the west, with $425 \mathrm{~km}$ shared borders. It is a non-industrialized province that is affected by dust phenomenon. Sampling sites in this study were limited to two cities located in Ilam Province, Ilam and Mehran (Figure 1). 


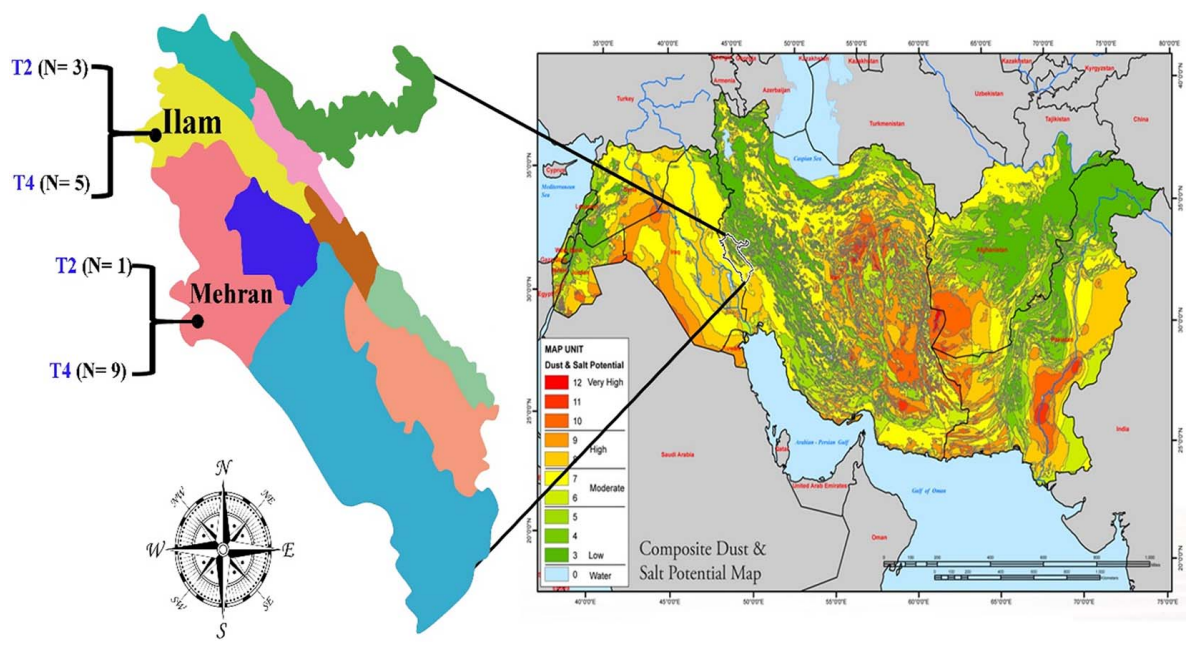

Figure 1. Sampling locations map in Ilam Province (map indicates composite dust and salt potential)

Sampling and Acanthamoeba culture

Fifty-five dust samples were collected in these regions and were dissolved in distilled sterile water and filtered using nitrocellulose filters (pore size: $0.45 \mu \mathrm{m}$; Sigma-Aldrich, USA). The filters were put onto $2 \%$ non-nutrient agar (Difco, USA) covered with trypticase-yeast extract-maltose and incubated at room temperature [14]. The plates were monitored daily for the presence of Acanthamoeba for up to a month. The observed trophozoites or cysts of Acanthamoeba were subcultured through transferring small pieces of agar with amoebae to a fresh plate to reduce fungal and bacterial contamination and increases Acanthamoeba to molecular assays.

\section{DNA extraction and PCR amplification assay}

DNA was extracted using a modified phenol-chloroform method [15]. To amplify the DF3 region of 18S rRNA (rDNA), specific primers JDP1 (forward: 5' GGC CCA GAT CGT TTA CCG TGA A 3') and JDP2 (reverse: 5' TCT CAC AAG CTG CTA GGG AGT CA 3') were used [16]. Amplification reactions were set for a total volume of $25 \mu \mathrm{l}$, containing $12.5 \mu \mathrm{l}$ Ampliqon (Taq DNA Polymerase Master Mix RED, Denmark), $1 \mu$ forward and reverse primers (10 pmol), $3 \mu \mathrm{l}$ DNA templates, and $8.5 \mu \mathrm{l}$ double-distilled water. PCR amplification was performed with a primary denaturing step at $94{ }^{\circ} \mathrm{C}$ for $1 \mathrm{~min}$, after 35 cycles at $94{ }^{\circ} \mathrm{C}$ for $35 \mathrm{~s}$, the annealing step was $56^{\circ} \mathrm{C}$ for $45 \mathrm{~s}$, and $72{ }^{\circ} \mathrm{C}$ for $1 \mathrm{~min}$. After this process, the final extension was performed at $72^{\circ} \mathrm{C}$ for $5 \mathrm{~min}$. 
Gel electrophoresis

Electrophoresis of PCR products was visualized using agarose gel electrophoresis on the $1 / 5 \%$ agarose gel (Invitrogen, Life Technologies GmbH, Germany) stained with safe stain.

Sequencing and genotyping of the isolates

PCR amplicons were sequenced at both directions, with PCR primers by Pouya Gostar Gene Company (www.pggene.com) in a Genetic Analyzer ABI 3730 (Bioneer, Daejeon, South Korea). The sequences analysis was performed with Bioedit Sequence Alignment Editor 7.1.3.0. Using the basic local alignment search tool (BLAST) analysis (http://blast.ncbi.nlm.nih.gov), sequencing data were aligned with Acanthamoeba genotype sequences available in the GenBank database, to determine the genotypes. Nucleotide sequences were submitted to the GenBank using BankIt.

\section{Thermotolerance and osmotolerance assays}

To predict the pathogenic potential of the positive isolates, thermal and osmotolerance tests are used currently. Osmotolerance and thermotolerance assays were developed as previously described [11]. Briefly, for the thermotolerance test, each positive strain was subcultured and incubated at two temperatures (37 and $44{ }^{\circ} \mathrm{C}$ ) for 24, 48, and $72 \mathrm{~h}$, and using D-Mannitol (Merck, Germany), each positive strain was subcultured in two plates at different molarities $(0.5$ and $1 \mathrm{M})$ for the osmotolerance assay. For both pathogenic tests, outgrowths of amoebae were checked by inverted microscopy $[11,17]$.

\section{Results}

Identification of Acanthamoeba isolates based on the morphological characteristics

Based on the morphological characteristics of amoebae, among the 55 dust samples, 18 (32.7\%) were positive for Acanthamoeba spp. Monoxenic culture was done by successive passages, such that only amoeba cysts and trophozoites remained in the cultures. Cysts are double-walled measuring 20-25 $\mu \mathrm{m}$ (Figure 2), and trophozoites have flat shape and spine-like structures (Figure 3), which illustrate them as related to the genus Acanthamoeba [2, 18]. The incidence of 


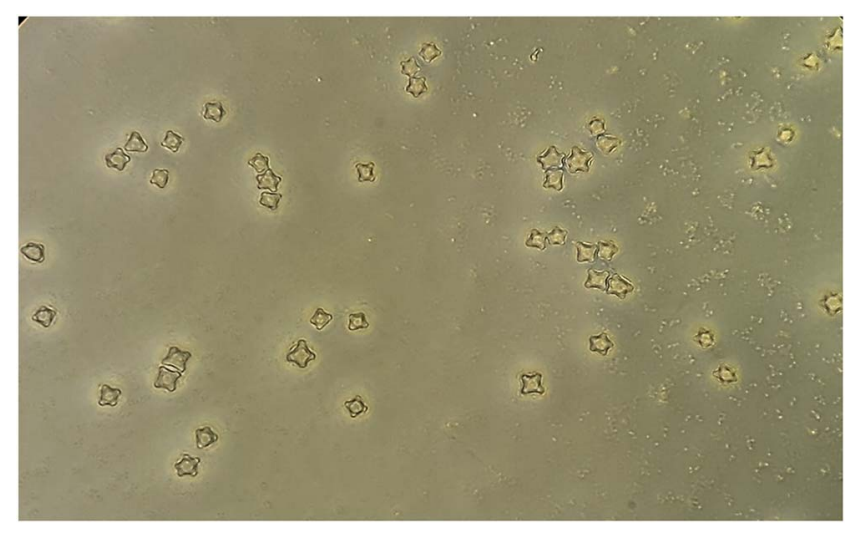

Figure 2. The image of Acanthamoeba cysts isolated from a dust sample in Ilam Province (magnification: 600×)

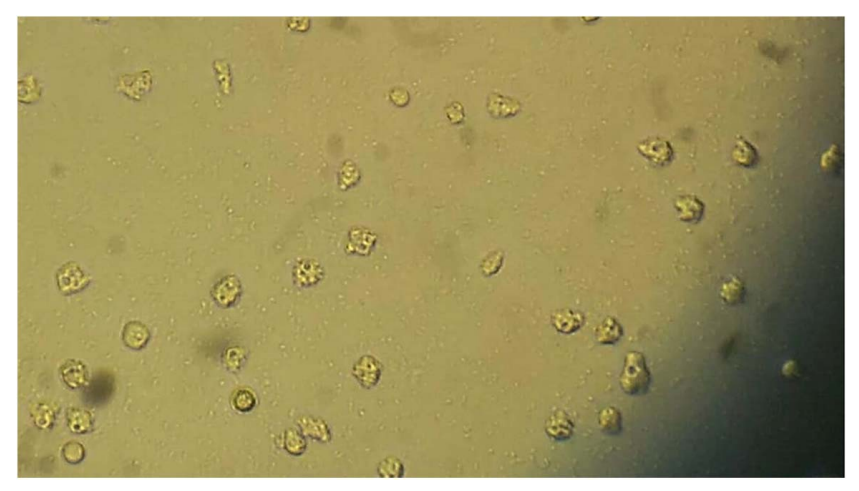

Figure 3. The image of Acanthamoeba trophozoites isolated from a dust sample in Ilam Province (magnification: $400 \times$ )

Acanthamoeba spp. was $26.6 \%$ (8 out of 30 samples) and $40 \%$ (10 out of 25 samples) in Ilam and Mehran cites, respectively. Eventually, 18 strains were submitted for molecular analysis.

Molecular analysis of Acanthamoeba isolates based on 18S rRNA gene

Acanthamoeba DNA was detected in all 18 morphological positive samples and was subjected to PCR amplification. A single approximately 460-bp product of the 18S rRNA gene was amplified in all the samples, which was consistent with the product size of Acanthamoeba genus. DNA sequence of the Acanthamoeba 
Table I. Data related to the positive strains of Acanthamoeba genotypes isolated from dust phenomenon in Ilam Province, Iran

\begin{tabular}{|c|c|c|c|c|c|}
\hline \multirow[b]{2}{*}{ Sampling site } & \multirow{2}{*}{$\begin{array}{l}\text { Culture/ } \\
\text { PCR }\end{array}$} & \multicolumn{2}{|c|}{ Thermo- and osmotolerance } & \multirow[b]{2}{*}{ Genotype } & \multirow[b]{2}{*}{ Acc. no. } \\
\hline & & $\left(37^{\circ} \mathrm{C} / 44{ }^{\circ} \mathrm{C}\right)$ & $0.5 \mathrm{M} / 1 \mathrm{M}$ & & \\
\hline Kodak Park & $+/+$ & $+/-$ & $+/-$ & $\mathrm{T} 2$ & MK192784 \\
\hline Public place & $+/+$ & $+/+$ & $+/+$ & $\mathrm{T} 4$ & MK192785 \\
\hline Imam Khomeini Hospital & $+/+$ & $-1-$ & $-1-$ & $\mathrm{T} 4$ & MK192786 \\
\hline Residential houses & $+/+$ & $+/-$ & $+/-$ & $\mathrm{T} 2$ & MK192787 \\
\hline Chaghasabz Park & $+/+$ & $+/+$ & $+/+$ & $\mathrm{T} 4$ & MK192788 \\
\hline School of Medicine & $+/+$ & $-1-$ & $-/-$ & $\mathrm{T} 4$ & MK192789 \\
\hline Residential houses & $+/+$ & $+/-$ & $+/-$ & $\mathrm{T} 2$ & MK192790 \\
\hline Qhaem Hospital & $+/+$ & $+/+$ & $+/+$ & $\mathrm{T} 4$ & MK192791 \\
\hline Health centers & $+/+$ & $+/+$ & $+/+$ & $\mathrm{T} 4$ & MK192792 \\
\hline Public place & $+/+$ & $-1-$ & $-1-$ & $\mathrm{T} 4$ & MK192793 \\
\hline Residential houses & $+/+$ & $+/-$ & $+/-$ & $\mathrm{T} 4$ & MK192794 \\
\hline Public place & $+/+$ & $+/+$ & $+/+$ & $\mathrm{T} 4$ & MK192795 \\
\hline Parvin Etesami High School & $+/+$ & $-1-$ & $-/-$ & $\mathrm{T} 2$ & MK192796 \\
\hline Mehran Park & $+/+$ & $+/-$ & $+/-$ & $\mathrm{T} 4$ & MK192797 \\
\hline Residential houses & $+/+$ & $+/-$ & $+/-$ & $\mathrm{T} 4$ & MK192798 \\
\hline Residential houses & $+/+$ & $+/-$ & $+/-$ & $\mathrm{T} 4$ & MK192799 \\
\hline Public place & $+/+$ & $+/+$ & $+/+$ & $\mathrm{T} 4$ & MK192800 \\
\hline Health centers & $+/+$ & $+/-$ & $+/-$ & $\mathrm{T} 4$ & MK192801 \\
\hline
\end{tabular}

Note: PCR: polymerase chain reaction.

isolates using BLASTn analysis revealed that 14 isolates $(77.7 \%)$ belonged to the T4 genotype, and 4 isolates $(22.3 \%)$ identified as T2 genotype under the accession numbers MK192784-MK192801 (Table I).

Pathogenesis of Acanthamoeba isolates based on tolerance assays

Six out of the 18 (38.8\%) Acanthamoeba strains were considered as highpotential pathogenic Acanthamoeba. These isolates could grow at high temperatures $\left(37\right.$ and $\left.44^{\circ} \mathrm{C}\right)$ and high osmolarity media $(1 \mathrm{M})$. Eight isolates demonstrated growth at $37^{\circ} \mathrm{C}$ and $0.5 \mathrm{M}$ osmolarity; hence, they are categorized as low-potential pathogens (Table I).

\section{Discussion}

Ilam Province due to arid and semi-arid climate having common borders with Iraq is one of the main centers of dust creation in Iran [19]. Dust is an 
atmospheric phenomenon that the frequency and intensity of this phenomenon in Ilam Province has been an upward trend. In the study of Maghsudi et al. [20], the highest concentration of total suspended particles and respirable particles (PM10) has been recorded 22.5 and $1.3 \mathrm{mg} / \mathrm{m}^{3}$ in Ilam city. In this study, we identified Acanthamoeba spp. in dust phenomenon of places that highly involved the human activities in dusty days.

Dust storms occur more in the summer and spring; therefore, during the summer, we collected 55 dust samples of dusty days. According to the results, 18 (32.7\%) out of 55 samples were positive for Acanthamoeba spp., as Niyyati et al. [15] also reported that the contamination of recreational and therapeutic geothermal water sources by Acanthamoeba in the Dehloran of Ilam Province was estimated to be $50 \%$, whereas in Ahvaz City, Southern Iran, the contamination of soil samples was estimated to be $26 \%$ [21].

To date, the phylogenetic analyses showed 21 genotypes (T1-T21 genotypes Acanthamoeba). In Iran, genotype T4 has the predominant Acanthamoeba genotypes and other Acanthamoeba genotype related to T2, T3, and T5 (Acanthamoeba lenticulata), T6, T9, T11, T13, and T15 (Acanthamoeba jacobsi) [22]. In this study, the genotyping data based on DF3 sequences revealed T4 and T2 genotypes, which are in agreement with the results of other researchers [23, 24]. The findings of this study revealed that the most common Acanthamoeba genotype in dust samples is the T4 genotype. In the study conducted by Mirjalali et al. [25], pathogenicity of Acanthamoeba T4 genotype was assessed via in vitro and in vivo tests. In this work, pathogenic potential of T4 genotypes in Iran was studied. In addition, in this study, this pathogenicity test showed that all of isolates were considered as high-potential pathogenic Acanthamoeba belonged to the T4 genotype. According to the literature, T4 genotype was the most commonly isolated genotype on human infections. More than $90 \%$ of keratitis cases were related to this genotype [26]. Although the number of T4 Iranian keratitis isolates was higher than that of T2 isolates $(61.5 \%$ vs. $23 \%)$, we determined T2 as the most genotype, followed by T4 [27]. Therefore, exposure to high concentrations of dust phenomenon in Ilam Province could be considered an important health hazard among the high-risk people. Eventually, public education is advisable to increase the consciousness among the high-risk individuals, such as contact lens users during a dusty day.

In conclusion, this study revealed Acanthamoeba related to T4 genotype as the most common strain dust phenomenon samples in Ilam Province. These strains could be a potential transmission for AK and high-risk people are exposed to them without being aware; therefore, considering health principles are suggested. 


\section{Acknowledgements}

This work was supported by a grant (no. 972001/100) from the Deputy of Research, Ilam University of Medical Sciences, Ilam, Iran. The authors are also grateful to Mr. Alireza Latifi for valuable comments.

RS designed all steps of the study and wrote the draft of the manuscript. RN made critical revisions and permitted the final version. RS and AN performed the experiments. All the authors reviewed and approved the final version of the manuscript.

\section{Conflict of Interest}

The authors declare no conflict of interest.

\section{References}

1. Marciano-Cabral, F., Cabral, G.: Acanthamoeba spp. as agents of disease in humans. Clin Microbiol Rev 16, 273-307 (2003).

2. Walochnik, J., Obwaller, A., Aspöck, H.: Correlations between morphological, molecular biological, and physiological characteristics in clinical and nonclinical isolates of Acanthamoeba spp. Appl Environ Microbiol 66, 4408-4413 (2000).

3. Khan, N. A.: Pathogenicity, morphology, and differentiation of Acanthamoeba. Curr Microbiol 43, 391-395 (2001).

4. dos Santos Gomes, T., Magnet, A., Izquierdo, F., Vaccaro, L., Redondo, F., Bueno, S., Sánchez, M. L., Angulo, S., Fenoy, S., Hurtado, C., Del Aguila, C.: Acanthamoeba spp. in contact lenses from healthy individuals from Madrid, Spain. PLoS One 11, e0154246 (2016).

5. Walochnik, J., Aichelburg, A., Assadian, O., Steuer, A., Visvesvara, G., Vetter, N., Aspöck, H.: Granulomatous amoebic encephalitis caused by Acanthamoeba amoebae of genotype T2 in a human immunodeficiency virus-negative patient. J Clin Microbiol 46, 338-340 (2008).

6. Kilvington, S., Price, J.: Survival of Legionella pneumophila within cysts of Acanthamoeba polyphaga following chlorine exposure. J Appl Bacteriol 68, 519-525 (1990).

7. Barker, J., Scaife, H., Brown, M. R.: Intraphagocytic growth induces an antibiotic-resistant phenotype of Legionella pneumophila. Antimicrob Agents Chemother 39, 2684-2688 (1995).

8. Corsaro, D., Köhsler, M., Di Filippo, M. M., Venditti, D., Monno, R., Di Cave, D., Berrilli, F., Walochnik, J.: Update on Acanthamoeba jacobsi genotype T15, including full-length 18S rDNA molecular phylogeny. Parasitol Res 116, 1273-1284 (2017).

9. Walochnik, J., Haller-Schober, E.-M., Kölli, H., Picher, O., Obwaller, A., Aspöck, H.: Discrimination between clinically relevant and no relevant Acanthamoeba strains isolated 
from contact lens-wearing keratitis patients in Austria. J Clin Microbiol 38, 3932-3936 (2000).

10. Booton, G. C., Visvesvara, G. S., Byers, T. J., Kelly, D. J., Fuerst, P. A.: Identification and distribution of Acanthamoeba species genotypes associated with non keratitis infections. J Clin Microbiol 43, 1689-1693 (2005).

11. Khan, N. A., Jarroll, E. L., Paget, T. A.: Molecular and physiological differentiation between pathogenic and nonpathogenic Acanthamoeba. Curr Microbiol 45, 197-202 (2002).

12. Khoshkish, A., Bohloul, A., Hejazizadeh, Z.: Synoptic analysis of dust storms in the Lorestan Province, Iran. J Geogr Sci 18, 91-110 (2011).

13. Ghasem, A., Shamsipour, A., Miri, M., Safarrad, T.: Synoptic and remote sensing analysis of dust events in Southwestern Iran. Nat Hazard 64, 1625-1638 (2012).

14. Ziaei Hezarjaribi, H., Nayeri Chegeni, T., Dadimoghadam, Y., Khoshzaban, F., Ghaffarifar, F., Fakhar, M.: Improved Acanthamoeba culture method using TYM medium. J Mazandaran Univ Med Sci 28, 156-160 (2018).

15. Niyyati, M., Saberi, R., Latifi, A., Lasjerdi, Z.: Distribution of Acanthamoeba genotypes isolated from recreational and therapeutic geothermal water sources in Southwestern Iran. Environ Health Insights 10, 69-74 (2016).

16. Booton, G. C., Kelly, D. J., Chu, Y. W., Seal, D. V., Houang, E., Lam, D. S. C., Byers, T. J., Fuerst, P. A.: 18S ribosomal DNA typing and tracking of Acanthamoeba species isolates from corneal scrape specimens, contact lenses, lens cases, and home water supplies of Acanthamoeba keratitis patients in Hong Kong. J Clin Microbiol 40, 1621-1625 (2002).

17. Kilic, A., Tanyuksel, M., Sissons, J., Jayasekera, S., Khan, N. A.: Isolation of Acanthamoeba isolates belonging to T2, T3, T4 and T7 genotypes from environmental samples in Ankara, Turkey. Acta Parasitologica 49, 246-522 (2004).

18. Niyyati, M., Sabari, R., Lorenzo-Morales, J., Salehi, R.: High occurrence of potentiallypathogenic free-living amoebae in tap water and recreational water sources in South-West Iran. Trop Biomed 33, 95-101 (2016).

19. Khaniabadi, Y. O., Daryanoosh, S. M., Amrane, A., Polosa, R., Hopke, P. K., Goudarzi, G., Mohammadi, M. J., Sicard, P., Armin, H.: Impact of middle eastern dust storms on human health. Atmos Pollut Res 8, 606-613 (2017).

20. Maghsudi, R., Kurd, N., Poornajaf, A.: Qualitative and quantitative evaluation of airborne microorganisms and the concentrations of particulate and respirable matter causing from dust storm in the air of Ilam City: Six-month cross sectional study. J Ilam Univ Med Sci 25, 60-69 (2017).

21. Rahdar, M., Niyyati, M., Salehi, M., Feghhi, M., Makvandi, M., Pourmehdi, M., Farnia, S.: Isolation and genotyping of Acanthamoeba strains from environmental sources in Ahvaz City, Khuzestan Province, Southern Iran. Iran J Parasitol 7, 22-26 (2012).

22. Spotin, A., Moslemzadeh, H. R., Mahami-Oskouei, M., Ahmadpour, E., Niyyati, M., Hejazi, S. H., Memari, F., Noori, J.: Phylogeography, genetic variability and structure of Acanthamoeba metapopulations in Iran inferred by $18 \mathrm{~S}$ ribosomal RNA sequences: A systematic review and meta-analysis. Asian Pac J Trop Med 10, 855-863 (2017).

23. Rahdar, M., Tavalla, M., Ebadi, S., Salehi, M.: The identification of free-living amoebae in water and soil from in and around Ahvaz, Southwest Iran. Biochem Cellular Arch 16, 379-382 (2016). 
24. Niyyati, M., Lorenzo-Morales, J., Rahimi, F., Motevalli-Haghi, A., Martín-Navarro, C. M., Farnia, S., Valladares, B., Rezaeian, M.: Isolation and genotyping of potentially pathogenic Acanthamoeba strains from dust sources in Iran. Trans R Soc Trop Med Hyg 103, 425-427 (2009).

25. Mirjalali, H., Niyyati, M., Abedkhojasteh, H., Babaei, Z., Sharifdini, M., Rezaeian, M.: Pathogenic assays of Acanthamoeba belonging to the T4 genotype. Iran J Parasitol 8, 530-535 (2013).

26. Khan, N. A.: Acanthamoeba: Biology and increasing importance in human health. FEMS Microbiol Rev 30, 564-595 (2006).

27. Maghsood, A. M., Sissons, J., Rezaian, M., Nolder, D., Warhurst, D., Khan, N. A.: Acanthamoeba genotype T4 from the UK and Iran and isolation of the T2 genotype from clinical isolates. J Med Microbiol 54, 755-759 (2005). 